\title{
Estudio multicéntrico de validación de una medición sencilla y reproducible para definir el centro de rotación de la cadera en la población mexicana
}

\section{Multicentric study of validation of a simple and reproductible measurement to define the hip center rotation in Mexican population}

\author{
Francisco Guillermo Castillo-Vázquez, ${ }^{*}$ Rodrigo Sánchez Rowold, ${ }^{\ddagger}$ \\ Roberto Berebichez-Fridman, $\$$ Jorge Ascencio Aragón $₫$
}

Citar como: Castillo-Vázquez FG, Sánchez RR, Berebichez-Fridman R, Ascencio AJ. Estudio multicéntrico de validación de una medición sencilla y reproducible para definir el centro de rotación de la cadera en la población mexicana. An Med ABC. 2021; 66 (2): 81-85. https://dx.doi.org/10.35366/100474

\section{RESUMEN}

Introducción: El centro de rotación de la cadera es un parámetro fundamental a restaurar en la artroplastia total de cadera, lo que permite optimizar su biomecánica, aumentando el tiempo de vida de los componentes protésicos. Objetivo: Validar y estandarizar un método sencillo, reproducible para determinar el centro de rotación de la cadera en la población mexicana. Material y métodos: Se evaluaron aleatoriamente 117 radiografías anteroposteriores de pelvis de adultos obtenidas en dos centros hospitalarios. Se realizó la determinación del centro de rotación de la cadera como el centro de un círculo concéntrico en la cabeza femoral y las líneas interisquiática, interiliaca y la perpendicular a las previas pasando por la porción más medial de la lágrima de Kohler (altura pélvica). Resultados: La altura pélvica media fue de

\section{ABSTRACT}

Introduction: The hip center rotation is a fundamental parameter which should be restored in hip arthroplasty. Reestablish it properly allows for the optimization of biomechanics and increases the lifespan of prosthetic components. Objective: Validate and standardize a simple and reproducible method for determining the hip center rotation in the Mexican population. Material and methods: We randomly evaluated 117 anteroposterior pelvic X-rays of adults obtained from two orthopedics centers. The hip center rotation was determined as the center of a concentric circle in the femoral head and three lines, interischiatic, interiliac and a perpendicular to previous passing through the medial part of the Kohler line (pelvic height). Results: The mean pelvic height was $247 \mathrm{~mm} \pm 13.38$

\footnotetext{
* Médico Especialista en Ortopedia y Traumatología.

₹ Médico Residente de Ortopedia y Traumatología de cuarto año.

$\S$ Médico Residente de Ortopedia y Traumatología de tercer año. Maestro en Ciencias Médicas.

" Médico Residente de Ortopedia y Traumatología de segundo año.
}

Centro Médico ABC. Ciudad de México, México.

Correspondencia: Dr. Francisco Guillermo Castillo-Vázquez E-mail: castillo.ortopedia@gmail.com
Recibido: 03/05/2021. Aceptado: 03/06/2021.

\section{Abreviaturas:}

$\mathrm{CRC}=$ Centro de rotación de la cadera

ATC $=$ Artroplastia total de cadera .

$\mathrm{AP}=$ Altura pélvica.

Lls = Líneas interisquiáticas.

Lli = Línea interiliaca.

RV-CRC = Relación vertical al centro de rotación de la cadera.

$\mathrm{RH}-\mathrm{CRC}=$ Relación horizontal al centro de rotación de la cadera. 
$247 \mathrm{~mm} \pm 13.38$ en hombres y $227 \mathrm{~mm} \pm 15.47$ en mujeres $(\mathrm{p}<$ 0.0001 ). La relación distancia horizontal fue de $16.63 \% \pm 1.76$ en hombres y $16.09 \% \pm 1.84$ en mujeres $(p=0.057)$, mientras que la relación distancia vertical en hombres fue de $30.9 \% \pm$ 2.91 y de $29.87 \% \pm 2.69$ en mujeres $(\mathrm{p}<0.05)$. Se demostró que existe una diferencia estadísticamente significativa entre los parámetros obtenidos en la población turca y los parámetros de la población mexicana así como entre sexos. Se definió como mediciones apropiadas una relación distancia horizontal de $16 \%$ en hombres y mujeres y una relación distancia vertical de $30 \%$ en hombres y $29 \%$ en mujeres para ser utilizadas en la población mexicana. Conclusiones: Definimos las medidas antropométricas específicas para establecer el centro de rotación en la población mexicana y las mediciones específicas con un método sencillo y reproducible, lo que aumenta la precisión de la planificación preoperatoria y mejora los resultados de pacientes sometidos a una artroplastia.

Palabras clave: Cadera, centro de rotación, población mexicana.

Nivel de evidencia: III

\section{INTRODUCCIÓN}

El centro de rotación de la cadera (CRC) se ha reconocido como un parámetro vital a restaurar al realizar una artroplastia total de cadera (ATC). Restaurar el CRC de forma adecuada optimiza el brazo de palanca que realizan los músculos abductores de la cadera, permite disminuir el estrés al que están sometidos los componentes protésicos aumentando su sobrevida, y ayuda a mantener una adecuada longitud de la extremidad en cuestión. Un método que ha demostrado de forma contundente mejorar la colocación del centro de rotación de la cadera es la planificación preoperatoria. ${ }^{1-3}$

Sin embargo, distintas patologías que afectan la anatomía nativa de la cadera como osteoartritis, displasia del desarrollo de la cadera o fracturas acetabulares, entre otras, ocasionan que establecer un adecuado CRC no sea siempre una tarea sencilla, ${ }^{4,5}$ por lo que se han propuesto varios métodos para definirlo, los cuales utilizan radiografías anteroposteriores de la pelvis y estructuras de referencia indirectas. Éstos pueden dividirse en métodos estadísticos, geométricos y basados en el movimiento de la cadera, cada uno con sus propias ventajas y desventajas. Entre las más importantes a tomar en cuenta están la complejidad de la medición, la dificultad de su reproducibilidad, su escasa difusión y no estar validada en nuestra población. . $^{6} 8$

Un método que demuestra ser sencillo y reproducible en comparación con otros reportados en la literatura para determinar el CRC es el descrito por el grupo de Bombaci, ${ }^{2}$ que utiliza la altura pélvica in men and $227 \mathrm{~mm} \pm 15.47$ in women $(p<0.0001)$. The ratio horizontal distance was $16.63 \% \pm 1.76$ in men and $16.09 \% \pm$ 1.84 in women $(p=0.057)$, while the vertical distance in men was $30.9 \% \pm 2.91$ and $29.87 \% \pm 2.69$ in women $(p<0.05)$. It was shown that there is a statistically significant difference between the parameters obtained in the Turkish population and the Mexican population, as well as between sexes. An horizontal distance ratio of $16 \%$ in men and women and a vertical distance ratio of $30 \%$ in men and $29 \%$ in women was established as the appropriate measurements to be used in the Mexican population. Conclusions: This study is the first to define the anthropometric basis of hip center rotation in the Mexican population and the specific measurements to be used using a simple and reproducible method, which will increase the accuracy of preoperative planning and improve the outcomes of patients undergoing a hip arthroplasty.

Keywords: Hip, hip center rotation, Mexican population.

Level of evidence: III

(AP) determinada por la distancia entre las líneas interisquiáticas (LIs) e interiliaca (LIi) y un porcentaje (30\% en hombres y $29 \%$ en mujeres) de esta altura hacia vertical de la LIs, relación vertical al centro de rotación de la cadera (RV-CRC) y un porcentaje de la AP hacia horizontal ( $14 \%$ en hombres y $13 \%$ en mujeres) de la lágrima de Kohler, relación horizontal al centro de rotación de la cadera ( $\mathrm{RH}-\mathrm{CRC})$, hasta el momento sólo validado en población turca. ${ }^{2,9}$

Debido a que está bien descrito que la morfología de la pelvis y cadera, y por lo tanto sus puntos de referencia pueden variar significativamente entre poblaciones, el propósito de este estudio es conocer si el método de Bombaci para obtener el CRC en otras poblaciones también es aplicable a la población mexicana.

El objetivo es validar y estandarizar un método sencillo y reproducible para determinar el CRC en la población mexicana.

\section{MATERIAL Y MÉTODOS}

Se realizó un estudio retrospectivo, observacional, descriptivo de corte transversal.

Utilizamos el teorema central del límite para determinar el tamaño de la muestra necesaria para obtener un resultado estadísticamente significativo con un nivel de confianza de $95 \%$ y un margen de error de $10 \%$ obteniendo un resultado de 97 muestras.

Se evaluaron de forma aleatoria 100 radiografías anteroposteriores de pelvis de adultos obtenidas en el Centro Médico ABC y 50 radiografías anteroposteriores de pelvis de adultos del Hospital «Dr. Germán Díaz Lombardo», descartando aquéllas con errores 

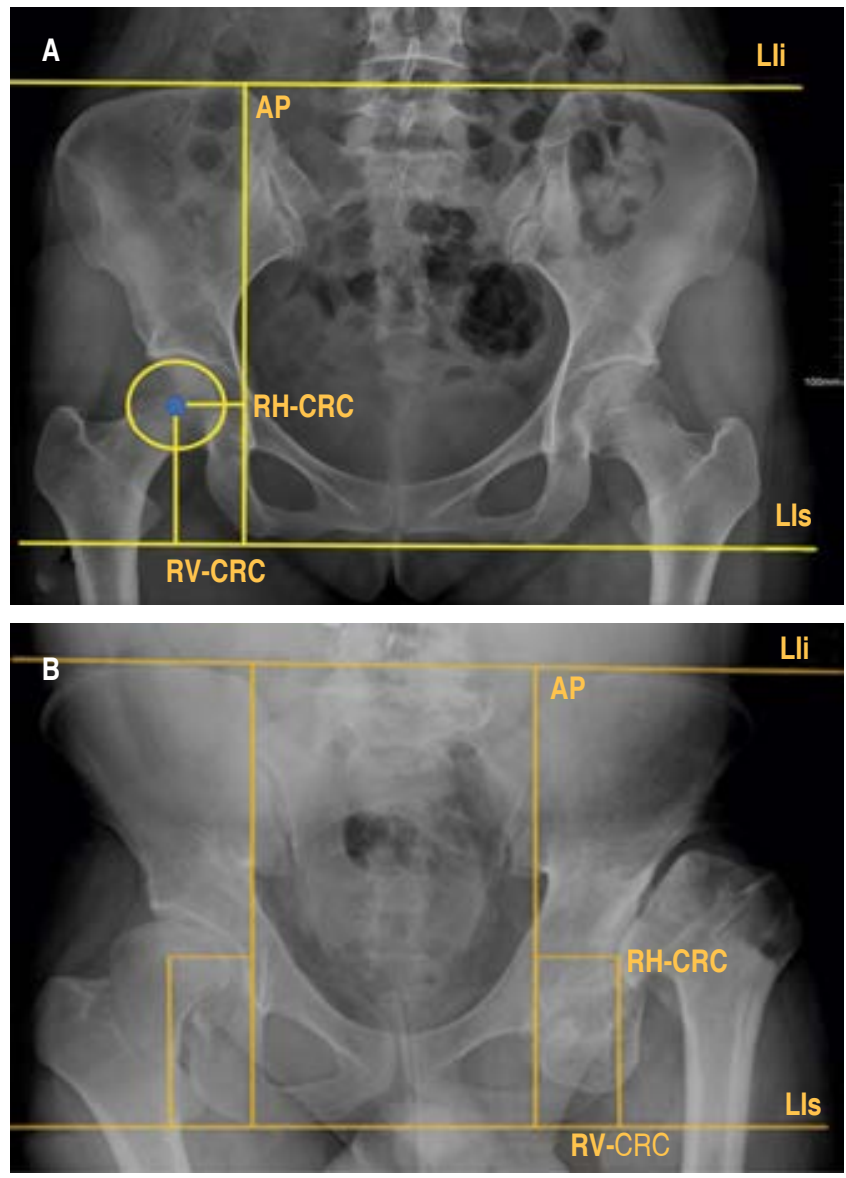

Figura 1: A) Se aprecian las medidas realizadas para definir el centro de rotación de la cadera (CRC) en una cadera sana. B) Se muestra un ejemplo del uso de esta medición para definir el CRC en una cadera con una deformidad anatómica importante.

$\mathrm{RH}-\mathrm{CRC}=$ relación de la distancia horizontal de la lágrima de Kohler al centro de rotación de la cadera; $\mathrm{RV}-\mathrm{CRC}$ = relación de la distancia vertical respecto al centro de rotación; Lli = línea interiliaca; Lls = línea interisquiática.

en la toma que pudieran afectar las mediciones. Se excluyeron las radiografías con el coxis y la sínfisis del pubis desalineados en los planos horizontal o vertical, con los agujeros obturadores asimétricos y/o con cirugías o padecimientos previos de las caderas.

Se obtuvo un total de 60 radiografías ( 28 mujeres y 32 hombres) para un total de 117 caderas sanas. Estas radiografías fueron evaluadas independientemente por dos de los autores, en éstas se replicaron todas las mediciones realizadas en el artículo original del grupo del Dr. Bombaci. Iniciamos determinando el CRC como el centro de un círculo concéntrico determinado por la cabeza femoral y su posición en la pelvis con tres referencias anatómicas; las LIs formadas por la conexión de los dos puntos más distales de las tuberosidades isquiáticas, la LIi formada por la conexión de los dos puntos más proximales de las crestas iliacas y una línea perpendicular a las previas pasando por la porción más medial de la lágrima de Kohler (altura pélvica, PH) (Figura 1). Todas las mediciones se realizaron en milímetros con el software digital Carestream (Carestream Health Inc., Nueva York, Estados Unidos).

Con base en estas referencias se midió la distancia entre el CRC determinado como el centro de la cabeza femoral y la porción más medial de las lágrimas de Kohler, lo que denominamos la distancia horizontal de la lágrima de Kohler al centro de rotación de la cadera (H-CRC) y posteriormente medimos la distancia entre el CRC y la línea LIs, lo que denominamos la distancia vertical respecto al centro de rotación (V-CRC).

Los resultados fueron analizados mediante la prueba estadística t de Student en el programa Microsoft Excel 2019 para compararlos con los resultados de diversas publicaciones internacionales.

\section{RESULTADOS}

Se replicaron las mediciones antropométricas utilizadas en el artículo original ${ }^{1}$ definiendo los valores de la AP así como la distancia vertical a la LIs (V-CRC) y horizontal desde la lágrima de Kohler hacia el centro de rotación (H-CRC) de cada radiografía. De acuerdo con estas mediciones la AP media fue de $247 \mathrm{~mm} \pm$ 13.38 en hombres y $227 \mathrm{~mm} \pm 15.47$ en mujeres $(p$ $<0.0001$ ), la distancia H-CRC de $41.3 \mathrm{~mm} \pm 4.68 \mathrm{en}$ hombres y $36.7 \mathrm{~mm} \pm 5.19$ en mujeres, mientras que la distancia V-CRC obtenida fue de $76 \mathrm{~mm} \pm 8.13 \mathrm{en}$ hombres y $68 \mathrm{~mm} \pm 6.91$ en mujeres.

Posteriormente se convirtieron la H-CRC y VCRC a porcentajes de la AP, de acuerdo con esto, la $\mathrm{RH}-\mathrm{CRC}$ fue de $16.63 \% \pm 1.76$ en hombres y $16.09 \%$ \pm 1.84 en mujeres cerca de presentar una diferencia estadísticamente significativa $(\mathrm{p}=0.057)$, mientras que la RV-CRC en hombres fue de $30.9 \% \pm 2.91$ y de $29.87 \% \pm 2.69$ en el grupo de mujeres, siendo esta diferencia estadísticamente significativa $\mathrm{p}<0.05$ ).

Debido a esta diferencia antropométrica entre hombres y mujeres comparamos, separando los datos entre sexos mediante la pruebas t de Student con el programa estadístico Microsoft Excel 2019, los resultados de nuestro trabajo con las poblaciones turcas masculina y femenina del artículo original (AP de $239 \pm 13.58 \mathrm{~mm}$ en hombres, $225 \pm 12.52 \mathrm{~mm}$ en mujeres ( $\mathrm{p}<0.0001)$, RH-CRC de $14.25 \% \pm 1.42$ en hombres y $13.69 \% \pm 1.38$ en mujeres y RV-CRC de 
$30.01 \% \pm 2.05$ y $29.10 \% \pm 2.35(\mathrm{p}=0.04)$, respectivamente), obteniéndose los resultados estadísticos que pueden apreciarse en la Tabla 1. En ésta se evidencia una diferencia estadísticamente significativa en la AP y RH-CRC, lo cual se traduce como una altura y distancia horizontal desde la lágrima de Kohler mayor en la población mexicana que en la turca en el grupo de hombres y en la RH-CRC en el grupo de mujeres, siendo este parámetro superior en las mujeres mexicanas.

\section{DISCUSIÓN}

Definir la posición adecuada del CRC en pacientes con alteraciones anatómicas ha sido un tema controversial y de debate. ${ }^{10,11} \mathrm{Si}$ bien existen referencias anatómicas bien establecidas para la colocación del CRC en una ATC, deformidades importantes pueden afectar su colocación. ${ }^{12,13}$ Los objetivos de la reconstrucción de la cadera mediante una ATC son: lograr un funcionamiento adecuado de las estructuras anatómicas circundantes, distribuir de forma adecuada las cargas para prevenir el desgaste prematuro de los componentes y obtener una adecuada estabilidad de la articulación. ${ }^{14,15}$ Es por esto que obtener una reconstrucción anatómica no sólo garantiza un mejor resultado, sino que disminuye el riesgo de requerir una cirugía de revisión. ${ }^{16,17}$ La planificación preoperatoria se ha convertido en un componente esencial para restablecer el CRC durante la ATC y se han descrito históricamente múltiples técnicas, por ejemplo, el método de Ranawat y el de Fessy. ${ }^{4,14}$ Estos métodos no están validados en nuestra población ni han demostrado ser reproducibles en la población general por diferencias antropométricas, además el hecho de que utilicen mediciones estándar que son altamente variables entre poblaciones, como demuestra nuestro estudio, pueden afectar su precisión al usarse en sujetos de razas diferentes a las que se utilizaron para definir la medición. ${ }^{1}$ Una desventaja de no lograr replicar el CRC es que la medialización del componente acetabular puede dar lugar a un aflojamiento temprano y con ello pérdida ósea pélvica, aumento del riesgo de fracturas periprotésicas y en consecuencia mayor probabilidad de requerir una cirugía de revisión con la morbilidad que ésta representa, mientras que por otro lado, la lateralización del componente femoral sobrecarga al aparato abductor de la cadera. ${ }^{1,3,17}$ En la actualidad se ha desarrollado un método aplicado a la población turca que ha demostrado ser sencillo y reproducible. ${ }^{1,2}$ Nuestro estudio es el primero del que tenemos conocimiento en el que se establecen las bases antropométricas del centro de rotación de la cadera en la población mexicana utilizando una población homogénea, multicéntrica y en un número suficiente para ser estadísticamente significativo. Al analizar las mediciones realizadas en esta población descubrimos que existen variaciones entre las medidas antropométricas de la población turca y la nuestra que deben tomarse en consideración una vez que se planifique la ATC, por lo tanto nos dimos a la tarea de definir porcentajes específicos para nuestra población para poder hacer uso del método de Bombaci (RH-CRC de $16 \%$ en hombres y mujeres y RV-CRC de $30 \%$ en hombres y $29 \%$ mujeres). Proponemos esta medición en nuestra población sobre otras descritas en la literatura por ser sencilla, reproducible y porque actualmente su uso está validado, lo que dará como resultado mayor precisión y por lo tanto, mejor funcionalidad en los pacientes, asimismo reducirá las complicaciones postoperatorias derivadas de una mala colocación.

\section{CONCLUSIONES}

Nuestro trabajo es el primer estudio mexicano del que tenemos conocimiento en establecer las bases antropométricas del centro de rotación de la cadera en una población heterogénea y suficiente para poder ser considerado como representativo de la población mexicana. Además, comparamos las mediciones obtenidas con las de la población turca ya definidas en la literatura, en las que se propuso una medición sencilla y reproducible para definir el centro de rotación de la cadera durante la planificación preoperatoria, apreciándose una diferencia estadísticamente significativa que nos obligó a establecer parámetros específicos para poder utilizar esta medición en nuestra población de forma eficaz. Proponemos el uso de

Tabla 1: Resultados estadísticos entre hombres y mujeres.

\begin{tabular}{|c|c|c|c|}
\hline & $\begin{array}{c}\text { Hombres vs. } \\
\text { mujeres* }\end{array}$ & Hombres $^{\star *}$ & Mujeres ${ }^{\star * *}$ \\
\hline Altura pélvica & 0.0001 & 0.0017 & 0.2802 \\
\hline $\mathrm{RH}-\mathrm{CRC}$ & 0.057 & 0.0001 & 0.0001 \\
\hline RV-CRC & 0.0495 & 0.002 & 0.0835 \\
\hline \multicolumn{4}{|c|}{$\begin{array}{l}\mathrm{RH}-\mathrm{CRC}=\text { relación de la distancia horizontal de la lágrima de Kohler al centro } \\
\text { de rotación de la cadera; RV-CRC = relación de la distancia vertical respecto } \\
\text { al centro de rotación. } \\
{ }^{*} \text { Hombres mexicanos vs. mujeres mexicanas. } \\
{ }^{* *} \text { Hombres mexicanos vs. hombres turcos. } \\
\text { ** Mujeres mexicanas vs. mujeres turcas. }\end{array}$} \\
\hline
\end{tabular}


esta medición en la población mexicana sobre otras descritas en la literatura por ser sencilla, reproducible y porque su uso está actualmente validado para nuestra población, lo que aumentará la precisión de la planificación preoperatoria y con ello mejorará la funcionalidad del paciente y reducirá la probabilidad de complicaciones postoperatorias como la morbimortalidad agregada que éstas conllevan.

\section{AGRADECIMIENTOS}

Los autores agradecen al Dr. Javier Camacho Galindo por su apoyo en la realización de este estudio.

\section{REFERENCIAS}

1. Lum ZC, Dorr LD. Restoration of center of rotation and balance of THR. J Orthop. 2018; 15 (4): 992-996.

2. Bombaci H, Simsek B, Soyarslan M, Murat Yildirim M. Determination of the hip rotation centre from landmarks in pelvic radiograph. Acta Orthop Traumatol Turc. 2017; 51 (6): 470-473.

3. Bicanic G, Delimar D, Delimar M, Pecina M. Influence of the acetabular cup position on hip load during arthroplasty in hip dysplasia. Int Orthop. 2009; 33 (2): 397-402.

4. O'Sullivan GS, Goodman SB, Jones HH. Computerized tomographic evaluation of acetabular anatomy. Clin Orthop Relat Res. 1992; (277): 175-1781.

5. Durand-Hill M, Henckel J, Satchithananda K, Sabah S, Hua J, Hothi $\mathrm{H}$ et al. Calculating the hip center of rotation using contralateral pelvic anatomy. J Orthop Res. 2016; 34 (6): 1077-1083.
6. Cassidy KA, Noticewala MS, Macaulay W, Lee JH, Geller JA. Effect of femoral offset on pain and function after total hip arthroplasty. J Arthroplasty. 2012; 27 (10): 1863-1869.

7. Bell AL, Pedersen DR, Brand RA. A comparison of the accuracy of several hip center location prediction methods. J Biomech. 1990; 23 (6): 617-621.

8. Nawabi DH, Meftah M, Nam D, Ranawat AS, Ranawat CS. Durable fixation achieved with medialized, high hip center cementless THAs for Crowe II and III dysplasia. Clin Orthop Relat Res. 2014; 472 (2): 630-636.

9. Kelley SS. High hip center in revision arthroplasty. J Arthroplasty. 1994; 9 (5): 503-510.

10. Zhang H, Zhou J, Liu Y, Guan J, Ding H, Wang Z et al. Midterm and long-term results of restoring rotation center in revision hip arthroplasty. J Orthop Surg Res. 2020; 15 (1): 152.

11. Pierchon F, Migaud H, Duquennoy A, Fontaine C. Evaluation radiologique du centre de rotation de la hanche. Rev Chir Orthop Reparatrice Appar Mot. 1993; 79 (4): 281-284.

12. Olmedo-Garcia N, Lopez-Prats F, Agullo A, Ortuño A, Palazon A. A comparative study of the accuracy of Ranawat's and Pierchon's methods to determine the centre of rotation in bilateral coxopathy. Skeletal Radiol. 2000; 29 (11): 652-655.

13. Lavy CB, Msamati BC, Igbigbi PS. Racial and gender variations in adult hip morphology. Int Orthop. 2003; 27 (6): 331-333.

14. Ranawat CS, Dorr LD, Inglis AE. Total hip arthroplasty in protrusio acetabuli of rheumatoid arthritis. J Bone Joint Surg Am. 1980; 62 (7): 1059-1065.

15. Patriquin ML, Steyn M, Loth SR. Metric analysis of sex differences in South African black and white pelves. Forensic Sci Int. 2005; 147 (2-3): 119-127.

16. Hirakawa K, Mitsugi N, Koshino T, Saito T, Hirasawa Y, Kubo T. Effect of acetabular cup position and orientation in cemented total hip arthroplasty. Clin Orthop Relat Res. 2001; (388): 135-142.

17. Sakalkale DP, Sharkey PF, Eng K, Hozack WJ, Rothman RH. Effect of femoral component offset on polyethylene wear in total hip arthroplasty. Clin Orthop Relat Res. 2001; (388): 125-134. 